\title{
A Radiology Hypertext System for Education and Clinical Decision Making
}

\author{
Charles E. Kahn, Jr
}

\begin{abstract}
Hypertext is a computer-based means of organizing information that allows one to explore the connections among related subjects. FACT/FILE is a hypertext computer system that provides access to a wide variety of information of interest to radiologists and radiology residents. Information is presented as notecards, or frames, which can contain textual information about relevant anatomy, physiology and pathology, as well as differential diagnosis "gamut" listings. More than $\mathbf{8 0 0}$ frames are available. Users can choose frames from an alphabetical index produced automatically from key words in each frame's title, or from a hierarchically organized subject index. Links between frames allow users to explore related frames and then return to the original frame. Physicians can add new frames to the system, so that specialized knowledge can be shared. This system has been implemented as a module of the radiology information system at the University of Chicago Medical Center.

Copyright $(1991$ by W.B. Saunders Company
\end{abstract}

KEY WORDS: hypertext, computer-aided instruction, radiological education, decision-support aids.

[T]he structures of ideas are not sequential. They tie together every whichway.'

$\mathbf{H}^{2}$ YPERTEXT is a computer-based method of organizing and presenting textual information, and has become popular as a learning tool and reference aid. It allows one to explore a topic by using the connections among related pieces of information. Hypertext computer systems present knowledge in nonlinear format, so that a reader can browse through the information or examine a specific topic in greater depth. ${ }^{1-3}$ Hypertext systems-and hypermedia systems, which integrate text, images, and sound-are beginning to find application in radiology. ${ }^{4-6}$ However, much of the clinical practice of radiology calls for a large amount of information that is not based on images, but on textual knowledge. The goal of this project was to develop and implement a hypertext system that would be simple to use and readily available to physicians in a clinical setting.

\section{SYSTEM DESIGN AND IMPLEMENTATION}

FACT/FILE is a hypertext system that provides an online reference file for radiologists and radiology residents. The system emulates a set of notecards, such as $5 \times 7$-inch paper index cards. Each notecard, or frame, consists of one computer screen of textual information about a specific topic, such as a disease entity or a radiologic differential diagnosis (gamut). Each frame contains up to 18 lines of text. The frame for Wormian bones is shown in Fig 1. At the bottom of the screen, the system displays the frame number, the author's initials, and the date on which the frame was created or last changed.

The advantage of a hypertext system is its ability to link related pieces of information. The links between frames allow users to move easily among related frames. The numbers within square brackets in the text indicate links to other frames. To select a linked frame, the user enters its number; the default is to return to the index. For example, in the frame on Wormian bones, the user can enter 2 to view the frame on cleidocranial dysostosis. If the user selects one of the links, the system will display that frame and allow the user to select another linked frame or, by default, return to the previous frame. In this way, the user can continue to move through the system's knowledge base. The system remembers the order in which the frames were chosen, so that the user can always go back to the previous frame.

Links can represent any type of connection between two frames. Frames that describe radiological manifestations of disease, such as Wormian bones, are linked to several of their causes, such as cleidocranial dysostosis. This frame is linked back to Wormian bones in a reciprocal, or two-way link. Many frames are linked because of a subset/superset relationship. For example, multiple sclerosis is a subset of demyelinating disease, which is in turn a subset of white-matter disease. Frames may be

From the Department of Radiology, University of Chicago, Chicago, $I L$.

Address reprint requests to Dr Charles E. Kahn, Jr, Department of Radiology, Medical College of Wisconsin, Milwaukee County Medical Complex, 8700 W Wisconsin Ave, Milwaukee, WI 53226.

Copyright $\odot 1991$ by W.B. Saunders Company 0897-1889/91/0404-0004\$03.00/0 
osteogenesis imperfecta [1]

cleidocranial dysostosis [2]

cretinism

idiopathic

Down syndrome [3]

hypophosphatasia

Menkes kinky-hair syndrome [4]

progeria [5]

pyknodysostosis [6]

$\# 426$ (CK $01 / 09 / 90)$ Go to $[0-6]:$ (index) //

Fig 1. Example frame: Wormian bones.

linked to contrast their features, such as pyknodysostosis and osteopetrosis. Associated disorders, such as von Hippel-Lindau syndrome and renal cysts, may be linked as well; no causal relationship is necessary. The frame for renal TB (Fig 2) shows several different types of links. An example of the breadth of linkage between topics is shown in Fig 3.

The frames are indexed by subject and title. The subject index is organized hierarchically: its first level contains a list of organ systems, such as "neuroendocrine system." Each subject entry can have one or more subentries. Currently, two of the topics (gastrointestinal system and genitourinary system) contain second-level entries. Table 1 lists the entries in the subject index and the number of frames indexed under each topic.
The system produces an alphabetical index from key words in each frame's title. From the main menu or from any subject index submenu, the user can search for a frame alphabetically by entering a word or part of a word. By entering only one letter, for example, the user can view all frames whose titles have words beginning with that letter. If the user has specified a subject, such as genitourinary system, then the search will include only those frames within that subject that meet the search criterion (Fig 4). The user can enter an asterisk to view the titles of all frames indexed by that topic. The titles of the frames are presented in a "key word in context" (KWIC) index format. Words such as "a," "of," and "the" are not indexed. This index is maintained automatically, and is modified

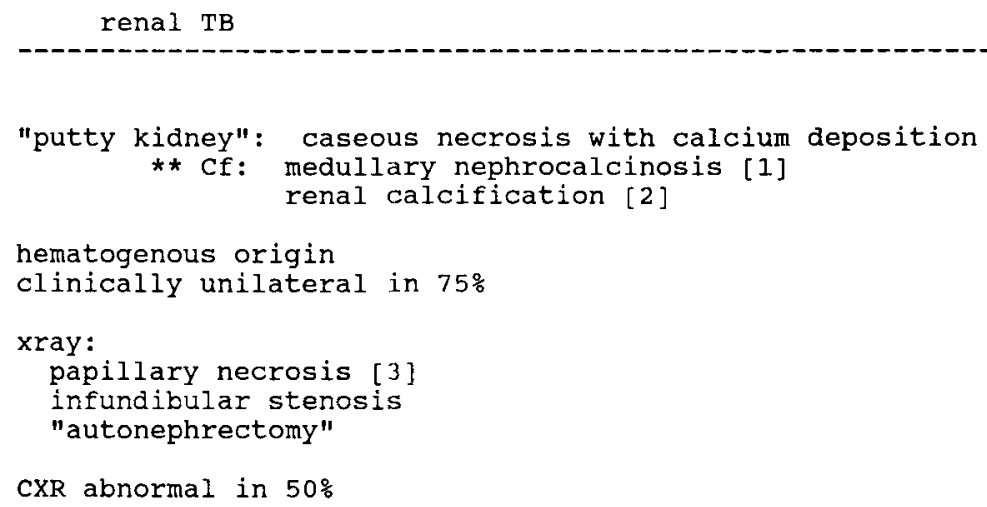


Fig 3. Network of links between frames.

each time a frame's title is changed or a new frame is added.

FACT/FILE also can select and present frames randomly, so that users can test their knowledge. While viewing a randomly selected frame, as with any frame, the user can select a linked frame, and then return to the original

Table 1. Number of Frames by Topic

\begin{tabular}{lc}
\hline \multicolumn{1}{c}{ Topic } & $\begin{array}{c}\text { No. of } \\
\text { Frames }\end{array}$ \\
\hline Neuroendocrine system & 119 \\
Cardiovascular system & 71 \\
Respiratory system & 81 \\
Gastrointestinal system & 189 \\
Esophagus & 29 \\
Stomach & 15 \\
Duodenum & 5 \\
Small bowel & 30 \\
Colon & 18 \\
Liver & 48 \\
Spleen & 10 \\
Pancreas & 17 \\
Other & 17 \\
Genito-urinary system & 114 \\
Breast & 8 \\
Adrenal & 13 \\
Kidney & 40 \\
Ureter & 7 \\
Bladder & 7 \\
Male genital tract & 9 \\
Female genital tract & 11 \\
Pregnancy/Fetus & 10 \\
Other & 916 \\
Musculoskeletal system & 27 \\
Multi-system entities & \\
Technical stuff/Miscellany & \\
Total & \\
\hline & \\
\hline &
\end{tabular}

one. The system continues to present frames randomly until the user directs it to return to the main menu.

FACT/FILE allows any user to add information into the system. In this way, physicians can use the system to share specialized knowledge. A user can create a new frame using a simple, interactive screen text editor. A link to another frame is represented by placing the number of the linked frame within square brackets in a line of text. A special option within the editing routine looks up the specified frame and inserts its number into the line of text. When displaying the frame, the system converts the numbers of the linked frames into serial numbers from 1 to 9.

An added frame is kept in its author's private collection, and is not available to other users. The frame's author can select it by title or by viewing his or her private index. FACT/FILE currently has three editors-this author and two radiology residents- - who can view and edit any frame in the system, including private frames. The editors can publish a frame by moving it from its author's private index into the general subject index. Once published in this manner, all FACT/FILE users can view the frame. Only the editors and the frame's author can change the frame's title or edit its text. Only the editors can change a frame's index code.

The programs are written in the ANSIstandard Massachusetts General Hospital Utility Multi-Programming System (MUMPS) language, a programming language designed for 


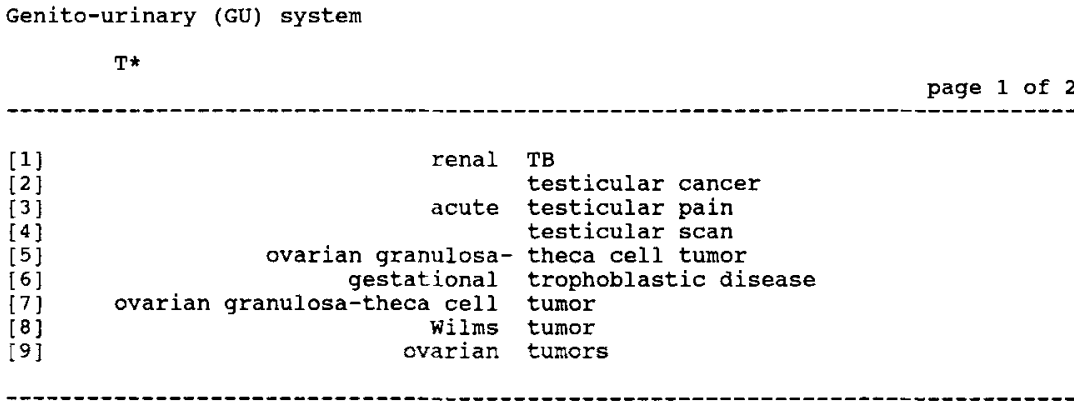

Select a topic (1-9): [more] //

biomedical database applications. FACT/FILE is available as a module of the MARS (ADAC Laboratories, Milpitas, CA) radiology information system at the University of Chicago Medical Center. ${ }^{7,8}$ This interactive, multiuser system is available to radiology department personnel at about 100 terminals throughout the department; it is used daily by radiologists and radiology residents. Information is retrieved and displayed instantly. Physicians can move easily from viewing a patient's examination history to exploring FACT/FILE's knowledge base.

The system currently contains 816 frames that discuss radiological gamuts and pertinent anatomy, physiology, and pathology. Of these frames, $498(61 \%)$ have one or more links to other frames (Table 2), some have as many as nine links. There are 1,022 links between frames; 590 of these are reciprocal (two-way) links where both frames are linked to each other. The entire knowledge base requires less than $500 \mathrm{kB}$ of storage.

\section{SYSTEM USE AND EVALUATION}

The system's use was studied from its initial implementation on January 11, 1990, to June 10, 1991, a period of 17 months (516 days). Potential users were informed about the sys-

\begin{tabular}{cc} 
Table 2. Number of Links Per Frame \\
\hline $\begin{array}{cc}\text { No. of } \\
\text { Links }\end{array}$ & $\begin{array}{c}\text { No. of } \\
\text { Frames }\end{array}$ \\
\hline 0 & 317 \\
1 & 238 \\
2 & 137 \\
3 & 58 \\
4 & 28 \\
5 & 18 \\
$\geq 6$ & 20 \\
& \\
Total & 816 \\
\hline
\end{tabular}

Fig 4. Alphabetic search within GU subindex.

tem's availability through the electronic mail feature of our radiology information system. Each time a FACT/FILE frame was viewed, the system recorded the frame number, date, time, user's ID, and the index or previous frame from which the frame was selected. In this period, the system was used a total of 11,003 times; an average of 21.3 frames were viewed per day. Residents were the most frequent users of the system; they accounted for more than $80 \%$ of the system's total use (Table 3).

Although the system was used on weekendsoften by the resident on call-most use occurred on weekdays, with greater use in the middle of the week (Fig 5). This distribution probably reflects the greater number of residents and faculty members in the department on weekdays. On almost $75 \%$ of the weekdays in the study period, the system was used by at least one person (Table 4). On more than one fourth of the weekdays, three or more people used the system. The maximum number of users in one day was 10 . The system remained unused on almost $75 \%$ of the Saturdays and Sundays. The system's use by hour is shown in Fig 6 . Here, too, the presence of a greater number of people between $8 \mathrm{AM}$ and $6 \mathrm{PM}$ may account for the increased system use during this time, although the system was used quite often in the evenings.

The most commonly viewed frames are listed in Table 5. Every frame was viewed at least once. No single frame accounted for more than $1 \%$ of total system use. Among all frames, the

Table 3. System Use

\begin{tabular}{lccrrr}
\hline \multirow{2}{*}{ Users } & $\begin{array}{c}\text { No. of } \\
\text { Users }\end{array}$ & $\begin{array}{c}\text { No. of } \\
\text { Frames Viewed }\end{array}$ & $\%$ of Use & Mean & Median \\
\cline { 2 - 6 } Residents & 23 & 8914 & 81.0 & 388 & 217 \\
Faculty & 15 & 1877 & 17.1 & 125 & 10 \\
Technical staff & 10 & 212 & 1.9 & 21 & 10 \\
\hline
\end{tabular}




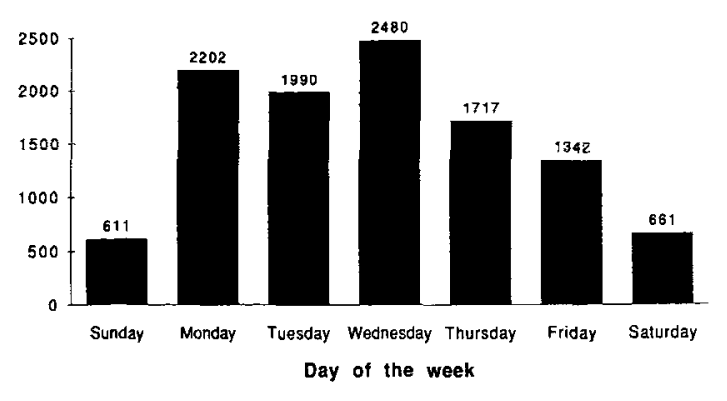

Fig 5. System use by day of week.

median number of times viewed was 10 . The links between frames were used extensively to explore the system's knowledge. Of the 11,003 frames viewed, $6,023(55 \%)$ were selected from either the alphabetical or topical index. The remainder were chosen via their links with other frames. More than $10 \%$ of the frames viewed were two or more links away from the originally selected frame. Users explored frames up to 21 links from the original frame.

The system's users reported a high level of satisfaction with the system. Fifteen physiciansthree staff radiologists and 12 radiology residents-evaluated the system (Table 6). Generally, they found that the system was easy to use and the on-line instructional help messages were adequate. They found the system useful for reviewing information and for learning new information. More than half of the participants reported a strong interest in adding their own frames to the system. Currently, five radiology residents have added a total of 30 frames to the system. They have indicated that the system is particularly useful for maintaining lists of differential diagnoses and for storing information about uncommon, eponymonic disorders.

Table 4. Number of Users Per Day

\begin{tabular}{cccc}
\hline \multirow{2}{*}{$\begin{array}{c}\text { No. of } \\
\text { Users per Day }\end{array}$} & Weekdays $(\%)$ & Weekends & Total \\
\cline { 2 - 4 } & $105(28.5)$ & $108(73.0)$ & 213 \\
1 & $93(25.3)$ & $32(21.6)$ & 125 \\
2 & $66(17.9)$ & $8(5.4)$ & 74 \\
3 & $42(11.4)$ & & 42 \\
4 & $29(7.9)$ & & 29 \\
$\geq 5$ & $33(9.0)$ & & 33 \\
& & & 516 \\
\hline
\end{tabular}

NOTE. The values in parentheses indicate the percentage of days on which there were the specified numbers of users.

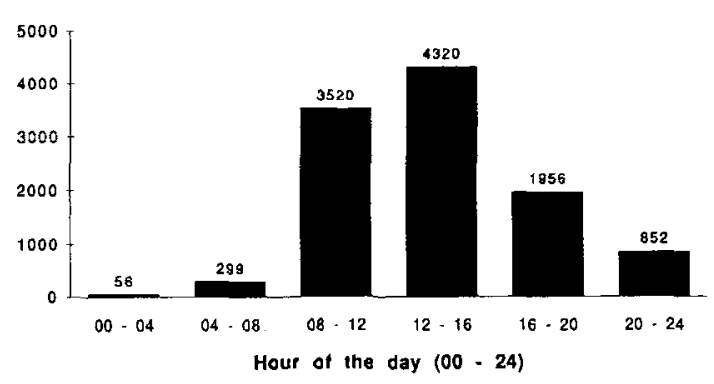

Fig 6. System use by hour.

\section{DISCUSSION}

Hypertext is particularly useful for demonstrating the interrelatedness of information. The hypertext format allows one to read text in a nonlinear fashion; one can digress and explore related topics, instead of proceeding in a regimented, page-by-page succession. The links between pairs of frames form a network of information, as shown in Fig 3. Such a network knits together seemingly disparate pieces of information. Users can traverse this network from any starting point to follow their curiosity or to answer specific questions. This network can transcend the usual-and often artificially rigid-divisions of knowledge into separate domains.

The system was used in three ways. First, as an educational aid, it was used extensively by residents and attending radiologists to browse the system's knowledge base. Much of this type of use involved the selection of frames from an alphabetical or topical index. Several residents indicated that they would select a topic and then view all frames within that topic. Second, the system was used extensively as a clinical

Table 5. The 12 Most Commonly Viewed Frames

\begin{tabular}{lcc}
\hline \multicolumn{1}{c}{ Topic } & $\begin{array}{r}\text { No. of } \\
\text { Times } \\
\text { Viewed }\end{array}$ & $\begin{array}{c}\text { Percent of } \\
\text { System } \\
\text { Use }\end{array}$ \\
\hline Pheochromocytoma & 65 & 0.59 \\
Aortic coarctation & 61 & 0.56 \\
Tuberous sclerosis (Bourneville disease) & 57 & 0.52 \\
Wormian bones & 54 & 0.49 \\
Abdominal aortic coarctation & 52 & 0.47 \\
Acro-osteolysis & 52 & 0.47 \\
Wilms' tumor & 48 & 0.44 \\
Demyelinating disease & 48 & 0.44 \\
Transposition of the great vessels & 44 & 0.40 \\
von Hippel-Lindau syndrome & 44 & 0.40 \\
Pyknodysostosis & 44 & 0.40 \\
MEN-1 (Wermer) syndrome & 44 & 0.40 \\
\hline
\end{tabular}


Table 6. Evaluation of the System by 15 Physicians

\begin{tabular}{|c|c|c|c|c|c|c|}
\hline \multirow[b]{2}{*}{ Question } & \multicolumn{6}{|c|}{ Response } \\
\hline & 1 & 2 & 3 & 4 & 5 & Mean \\
\hline Easy to use & 0 & 1 & 0 & 6 & 8 & 4.40 \\
\hline Adequate "help" messages & 0 & 2 & 1 & 6 & 6 & 4.07 \\
\hline Appropriate level of information & 0 & 0 & 2 & 10 & 3 & 4.07 \\
\hline Rapid access to information & 0 & 2 & 0 & 4 & 9 & 4.33 \\
\hline Cross-links between notecards useful & 0 & 1 & 0 & 1 & 13 & 4.73 \\
\hline Useful for learning new information & 0 & 0 & 1 & 4 & 10 & 4.60 \\
\hline Useful for reviewing information & 0 & 0 & 1 & 6 & 8 & 4.47 \\
\hline Would add notecards to the system & 0 & 3 & 2 & 2 & 8 & 4.00 \\
\hline
\end{tabular}

Key: 1, strongly disagree; 2 , disagree; 3 , uncertain or no opinion; 4 , agree; 5 , strongly disagree.

tool. Several physicians, particularly residents, used the system frequently to answer specific clinical questions and to help formulate differential diagnoses. Of the 23 residents who used the system, 18 used it on more than one occasion. Twelve residents used it on 20 or more separate occasions. Finally, the system was used as a personal notepad, where physicians recorded their own mnemonic aids, associations, and notes.

FACT/FILE allows users to work together to share knowledge. Any user can add frames to the system and link them to existing frames. New frames created by a user are kept in a private library and are accessible only to the person who created them. They are not placed in the general index until one of the system's editors gives approval. The editors provide a form of peer review to prevent inaccurate information from being disseminated. By sharing knowledge through a hypertext system, physicians can work together to improve medical education and clinical practice. Software for computer-supported cooperative work, called groupware, is an emerging discipline in computer science and ergonomics. ${ }^{9}$ A multiuser hypertext system has been described. ${ }^{10}$

FACT/FILE is a simple, MUMPS-based, multiuser hypertext system that can be integrated into a clinical radiology information system. MUMPS is a common language for biomedical database applications, and forms the basis of several widely-disseminated radiology information systems, such as MARS, DECrad (Digital Equipment Corp, Maynard, MA) and Maxifile (Dimensional Medicine, Minnetonka, MN). Because the user identification codes are the only part of FACT/FILE specific to the MARS system, it should be simple to adapt the system to other MUMPS-based information systems.

Hypertext holds great promise in both education and medical practice. Medical students and physicians can extract information easily from the system and direct their own learning. The system offers a rapid, interactive reference aid for clinical practice that displays radiologic gamuts and detailed descriptions of various diseases. Because physicians can explore FACT/ FILE's knowledge without leaving their work area, the system is useful as a desktop reference and an educational tool, particularly for radiology residents.

\section{ACKNOWLEDGMENT}

I thank Richard E. Sayre for valuable discussions, and Leslie Cleveland for secretarial assistance.

\section{REFERENCES}

1. Nelson TE: Computer Lib/Dream Machines. South Bend, IN, The Distributors, 1974, p DM44 ff

2. Nelson TE: The hypertext. Proceedings of the World Documentation Federation, 1965 in Computer Lib/Dream Machines. South Bend, IN, The Distributors, 1974

3. Rubeck RF: Hypertext programs for computerassisted learning. Acad Med 66:193, 1991

4. Jaffe CC, Lynch PJ, Smeulders AWM: Hypermedia techniques for diagnostic imaging instruction: videodisk echocardiography encyclopedia. Radiology 171:475-480, 1989

5. Chen CC, Hoffer PB, Swett HA: Hypermedia in radiology: computer-assisted education. J Digital Imaging 2:48-55, 1989

6. Hennessey JG, Fishman EK, Kuhlman JE, et al:
Computer-based learning in radiology: a hypermedia application in CT. AJR 155:1317-1320, 1990

7. Lehr JL, Lodwick GS, Nicholson BF, et al: Experience with the MARS (Missouri Automated Radiology System). Radiology 106:289-294, 1973

8. Lehr JL: Installation of MARS II at the University of Chicago, in: Proceedings of the Eighth Conference of Computer Applications in Radiology. Chicago, IL American College of Radiology, 1984, pp 35-46

9. Greenberg S: Computer-supported cooperative work and groupware: an introduction to the special issues. Int $\mathrm{J}$ Man-Machine Studies 34:133-141, 1991

10. Rein GL, Ellis CA: rIBIS: a real-time group hypertext system. Int J Man-Machine Studies 34:349-367, 1991 